\title{
Óleo essencial de Baccharis trimera (Less.) DC. sobre o potencial fisiológico de sementes de feijão caupi
}

\author{
XAVIER, M.V.A.; BRITO, S.S.S.; OLIVEIRA*, C.R.F.; MATOS, C.H.C.; PINTO, M.A.D.S.C. \\ Unidade Acadêmica de Serra Talhada, Universidade Federal Rural de Pernambuco, (UASTIUFRPE). Fazenda \\ Saco, s/n, Caixa Postal 063, CEP: 56900-000, Serra Talhada-Brasil *crfoliveira@hotmail.com
}

\begin{abstract}
RESUMO: O presente trabalho teve como objetivo avaliar o efeito alelopático do óleo essencial de plantas de carqueja, Baccharis trimera (Less.) DC., sobre a germinação de sementes de feijão Vigna unguiculata (L.) Walp. Foi avaliado o efeito do óleo essencial de B. trimera sobre V. unguiculata nas dosagens $20 \mu \mathrm{L}, 15 \mu \mathrm{L}, 10 \mu \mathrm{L}, 5 \mu \mathrm{L}$ e testemunha. A qualidade fisiológica das sementes foi determinada pela porcentagem de emergência, velocidade de emergência e índice de velocidade de emergência. O delineamento experimental foi o inteiramente casualizado, em esquema fatorial $5 \times 2$ com cinco repetições. Não foi observado efeito inibidor do óleo essencial de $B$. trimera na germinação de sementes de feijão caupi, caracterizando-se como de efeito alelopático benéfico. De acordo com os resultados obtidos, o óleo essencial de $B$. trimera revelouse eficiente na manutenção da viabilidade dessas sementes.
\end{abstract}

Palavras-chave: Vigna unguiculata, Baccharis trimera, óleo essencial, potencial alelopático, viabilidade de sementes

\begin{abstract}
The essential oil effects of Baccharis trimera (Less.) DC. on the physiological potential of cowpea bean seeds. This research aimed to evaluate the allellopathic effect of essential oils of the Baccharis trimera (Less.) DC. plants on the seeds germination of cowpea Vigna unguiculata (L.) Walp. The effect of $B$. trimera essential oil on $V$. unguiculata was evaluated at levels of $20 \mu \mathrm{L}, 15 \mu \mathrm{L}, 10 \mu \mathrm{L}, 5 \mu \mathrm{L}$ and control. The physiologic quality of the seeds was determined by percentage emergence, the rate of speed emergence and speed emergence index. The data analysis was carried out using an entirely randomized design, in a $5 \times 2$ factorial scheme with five repetitions. An inhibitory effect of $B$. trimera essential oil on bean seeds germination was not observed, which is characterized as a beneficial allelopathic effect. Based on the results, the $B$. trimera essential oil proved efficient in the viability maintenance of these seeds.
\end{abstract}

Key words: Vigna unguiculata, Baccharis trimera, essential oil, allellopathic potential, seeds viability

\section{INTRODUÇÃO}

O feijão-de-corda ou feijão caupi (Vigna unguiculata (L.) Walp.) possui grande importância agronômica e econômica por ser um dos grãos mais consumidos no Brasil, servindo de base para a preparação de diversos pratos da culinária brasileira.

Segundo a FAO (2009), 12,5 milhões de hectares são cultivados com feijão caupi no mundo e destes, oito milhões estão na África (Almeida et al., 2009). Essa espécie apresenta algumas características técnicas que facilitam o cultivo, como ciclo curto, resistência ao stress hídrico, baixa exigência nutricional e elevada adaptabilidade a solos arenosos.

O Brasil é um dos maiores produtores de feijão-caupi, cuja produção historicamente concentra- se nas regiões Nordeste (1,2 milhão de hectares) e Norte (55,8 mil hectares) do país, contribuindo com $35,6 \%$ da área plantada e $15 \%$ da produção de feijão total (feijão-caupi + feijão-comum). Entretanto, a cultura está conseguindo espaço também na região Centro-Oeste, em razão do desenvolvimento de cultivares com características que favorecem o cultivo mecanizado (Silva, 2009).

A avaliação agronômica do feijão caupi, bem como a conservação em médio e longo prazo é de suma importância para a sustentabilidade da cultura (Wetzel et al., 2006). Tanto o armazenamento como o tratamento de sementes tornam-se práticas de extrema importância para a manutenção da germinação e do vigor das sementes, contribuindo

Recebido para publicação: setembro de 2011

Aceito para publicação: março de 2012

Rev. Bras. PI. Med., Botucatu, v.14, n.esp., p.214-217, 2012. 
para uma produtividade satisfatória (Machado, 2000). No entanto nas regiões tropicais o armazenamento é uma das principais limitações encontradas devido à influência de diversos fatores na manutenção da viabilidade das sementes, como qualidade fisiológica inicial da semente, condições climáticas durante a maturação, grau de umidade, temperatura de armazenamento e, principalmente, ação de microrganismos e insetos (Carvalho \& Nakagawa, 2000).

Diversas pesquisas têm demonstrado a viabilidade do uso de compostos bioativos obtidos de plantas no controle de insetos-praga de grãos armazenados, principalmente pela fácil aquisição, baixo custo, eficiência e segurança para os aplicadores e consumidores (Shaaya et al., 1997). Geralmente esses compostos podem ser utilizados na forma de pó, extrato aquoso ou orgânico, óleos essenciais e óleos emulsionáveis. A eficiência e a toxicidade dos óleos essenciais no tratamento de sementes são influenciadas por diversos fatores, dentre eles, a composição química.

Algumas espécies vegetais já foram testadas e revelaram-se bastante promissoras no controle de insetos-praga de feijão comum armazenado, como Tagetes minuta L., Ocimum canum Sims e Cinnamomum zeylanicum Ness (Weaver et al. 1994a; 1994b).

Baccharis trimera Less. (DC.), conhecida popularmente como carqueja, é uma planta que ocorre espontaneamente em terrenos baldios, podendo ser considerada planta daninha. Não possui folhas verdadeiras, as flores são brancoamareladas e surgem em pequenos capítulos. Os usos populares da espécie estão geralmente relacionados ao sabor extremamente amargo (PioCorrêa, 1994; Mors et al., 2000).

Estudos têm demonstrado que $B$. trimera é uma planta que possui princípios ativos com várias propriedades, sendo utilizada amplamente com fins terapêuticos (Bona et al., 2002). Além disso, pode apresentar efeito alelopático, a partir dos extratos aquosos, sobre sementes de algumas plantas, como o tomate (Castro \& Ferreira, 2001) e o alface (Fritz et al., 2007), dentre outros.

$O$ presente trabalho foi realizado com 0 objetivo de verificar os efeitos do óleo essencial de $B$. trimera sobre o potencial fisiológico das sementes de feijão caupi, cv IPA 206, tratadas com esse óleo por meio dos processos de fumigação e impregnação.

\section{MATERIAL E MÉTODO}

O experimento foi conduzido na Universidade Federal Rural de Pernambuco - Unidade Acadêmica de Serra Talhada, realizado em bandejas dispostas sobre uma bancada a 1,00 m do solo e sombreadas a $50 \%$, à temperatura ambiente $\left(32,5^{\circ} \mathrm{C}\right)$ no município Serra Talhada-PE. Foram utilizadas sementes de feijão-caupi, cultivar IPA 206, tratadas com o óleo essencial de $B$. trimera.

Foram utilizadas quatro dosagens $(20 \mu \mathrm{L}, 15$ $\mu \mathrm{L}, 10 \mu \mathrm{L}$ e $5 \mu \mathrm{L}$ ), além da testemunha (sem aplicação de óleo), sendo utilizadas duas formas de aplicação do óleo. Na primeira aplicação as sementes foram diretamente impregnadas com o óleo essencial no interior de recipiente plástico, com o auxílio de pipetador automático, mediante agitação manual durante dois minutos. Na segunda aplicação utilizouse câmaras de fumigação (adaptadas de Aslan et al., 2004), compostas de recipientes de vidro de 2,5 L de capacidade onde as sementes não tiveram contato direto com o óleo. Neste caso tiras de papel de filtro de $5 \times 2 \mathrm{~cm}$ foram impregnadas com o óleo de $B$. trimera, com o auxílio de pipetador automático, sendo fixadas na superfície inferior da tampa dos recipientes. A semeadura foi realizada 24 horas após o tratamento das sementes com o óleo essencial, sendo utilizadas bandejas de isopor com 200 células com substrato para hortaliças. Colocou-se uma semente por célula na profundidade de aproximadamente $3 \mathrm{~cm}$, sendo realizadas irrigações diárias.

Foi empregado o delineamento inteiramente casualizado, em esquema fatorial $5 \times 2$ (cinco dosagens do óleo essencial $x$ duas formas de aplicação), com cinco repetições de 20 sementes cada.

Foram realizadas contagens diárias do número de plântulas emersas desde o segundo dia após a semeadura até o 8o dia. Avaliou-se a porcentagem de emergência (\%E), a velocidade de emergência (VE), o índice de velocidade de emergência (IVE) e o coeficiente de velocidade de germinação (CVE); empregando-se as seguintes fórmulas:

a) Velocidade de emergência (Edmond \& Drepala, 1958):

Em

$$
\mathrm{VE}=\left(\mathrm{N}_{1} \mathrm{E}_{1}\right)+\left(\mathrm{N}_{2} \mathrm{E}_{2}\right)+\ldots+\left(\mathrm{N}_{\mathrm{n}} \mathrm{E}_{\mathrm{n}}\right) / \mathrm{E}_{1}+\mathrm{E}_{2}+\ldots+
$$

Onde: VE = velocidade de emergência (dias); $\mathrm{E}=$ número de plântulas emergidas em cada repetição; $\mathrm{N}$ = número de dias da semeadura à primeira, à segunda e à última contagem.

b) Índice de velocidade de emergência (Maguire, 1962):

IVE $=E_{1} / N_{1}+E_{2} / N_{2}+\ldots+E_{n} / N_{n}$

Onde: IVE = Índice de velocidade de emergência; $E$ = número de plântulas emergidas em cada repetição; $\mathrm{N}$ = número de dias da semeadura à primeira, à segunda e à última contagem.

c) Coeficiente de velocidade de emergência (Furbeck et al., 1993):

$$
\text { CVE }=\mathrm{E}_{1}+\mathrm{E}_{2}+\ldots+\mathrm{Em} \times 100
$$

$$
\left(\mathrm{N}_{1} \mathrm{E}_{1}\right)+\left(\mathrm{N}_{2} \mathrm{E}_{2}\right)+\ldots+\left(\mathrm{N}_{\mathrm{n}} \mathrm{E}_{\mathrm{n}}\right)
$$

Onde: $\mathrm{CVE}=$ Coeficiente de velocidade de 
emergência; $E$ = número de plântulas emergidas em cada repetição; $\mathrm{N}$ = número de dias da semeadura à primeira, à segunda e à última contagem.

Os resultados foram submetidos à análise de variância, sendo as médias comparadas pelo teste de Tukey ao nível de $5 \%$ de probabilidade (Canteri et al., 2001).

\section{RESULTADO E DISCUSSÃO}

Não foram observadas diferenças significativas para a germinação de sementes de V. unguiculata tratadas com o óleo essencial de B. trimera em diferentes dosagens (Figura 1).

De acordo com Lobato et al. (2007), o óleo essencial de Piper aduncum não apresentou respostas fitotóxicas sobre a germinação de sementes de $V$. unguiculata, em altas concentrações. Da mesma forma, Medeiros et al. (2007) observaram que o uso de folhas de Azadirachta indica ( $A$. Juss) também não apresentou efeitos sobre a porcentagem de germinação de sementes de caupi.

Em relação ao tipo de aplicação do óleo essencial de $B$. trimera (fumigação ou impregnação), para os dois métodos também não foram observadas diferenças significativas (Tabela 1). Assim, observou-se que o óleo essencial não interferiu na germinação do feijão caupi.

Observou-se que não houve diferenças estatísticas para as variáveis avaliadas, índice de velocidade de emergência, velocidade de emergência e coeficiente de velocidade de emergência (Tabela 2).

Neste caso, tanto as sementes não-tratadas (Testemunha) quanto às submetidas ao óleo essencial

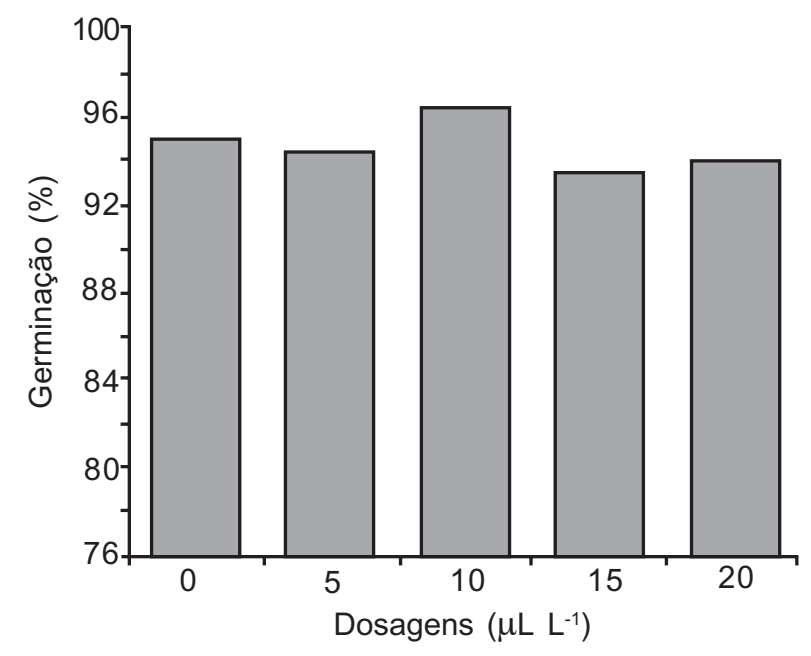

FIGURA 1. Efeito de diferentes dosagens do óleo essencial de $B$. trimera sobre a germinação (emergência) de sementes de $V$. unguiculata.
TABELA 1. Porcentagem de germinação (emergência) de sementes de $V$. unguiculata submetidas ao óleo essencial de $B$. trimera, sob diferentes aplicações.

\begin{tabular}{lc}
\hline Método de Aplicação & Germinação \\
\hline Fumigação & $94,75 \mathrm{~A}$ \\
Impregnação & $95,00 \mathrm{~A}$ \\
Testemunha & $94,00 \mathrm{~A}$ \\
\hline
\end{tabular}

*Médias seguidas de mesma letra, nas colunas, não diferem entre si significativamente pelo teste de Tukey, a $5 \%$ de probabilidade.

TABELA 2. Médias estimadas da velocidade de emergência (VE), índice de velocidade de emergência e coeficiente de velocidade de emergência de sementes de $V$. unguiculata tratadas com óleo essencial de $B$. trimera sob diferentes dosagens e formas de aplicação.

\begin{tabular}{|c|c|c|c|}
\hline $\begin{array}{l}\text { Método de Aplicação } \\
\text { e Dosagens }\end{array}$ & $\begin{array}{c}\text { IVE } \\
\text { (Dias) }\end{array}$ & $\begin{array}{l}\text { VE } \\
\text { (Dias) }\end{array}$ & $\begin{array}{l}\text { CVE } \\
(\%)\end{array}$ \\
\hline \multicolumn{4}{|l|}{ Fumigação } \\
\hline $5 \mu \mathrm{L}$ & $3,44 a$ & $5,90 \mathrm{a}$ & $17,32 \mathrm{a}$ \\
\hline $10 \mu \mathrm{L}$ & 3,62 a & $5,63 a$ & $18,42 \mathrm{a}$ \\
\hline $15 \mu \mathrm{L}$ & $3,76 \mathrm{a}$ & $5,19 a$ & $19,24 \mathrm{a}$ \\
\hline $20 \mu \mathrm{L}$ & 3,58 a & $5,43 a$ & $18,54 \mathrm{a}$ \\
\hline Testemunha & 3,51 a & $5,04 a$ & $19,58 \mathrm{a}$ \\
\hline Impregnação & $\begin{array}{l}\text { IVE } \\
\text { (Dias) }\end{array}$ & $\begin{array}{l}\text { VE } \\
\text { (Dias) }\end{array}$ & $\begin{array}{l}\text { CVE } \\
(\%)\end{array}$ \\
\hline $5 \mu \mathrm{L}$ & $3,46 a$ & $5,94 \mathrm{a}$ & $17,22 \mathrm{a}$ \\
\hline $10 \mu \mathrm{L}$ & $3,71 \mathrm{a}$ & $5,27 \mathrm{a}$ & $18,96 \mathrm{a}$ \\
\hline $15 \mu \mathrm{L}$ & $3,79 a$ & $5,21 \mathrm{a}$ & $19,20 \mathrm{a}$ \\
\hline $20 \mu \mathrm{L}$ & $3,31 \mathrm{a}$ & $5,81 \mathrm{a}$ & $17,50 \mathrm{a}$ \\
\hline Testemunha & $3,61 a$ & $5,04 a$ & $19,82 \mathrm{a}$ \\
\hline
\end{tabular}

*Médias seguidas de mesma letra, nas colunas, não diferem entre si significativamente pelo teste de Tukey, a $5 \%$ de probabilidade.

de B. trimera apresentaram o mesmo padrão, independentemente das dosagens e dos métodos utilizados (fumigação ou impregnação). Estes resultados indicam que este óleo não apresenta efeito alelopático significativo em sementes de $V$. unguiculata nas dosagens utilizadas no presente estudo.

O efeito alelopático de extratos de carqueja já foi constatado em sementes de outras plantas, como é o caso do picão-preto (Bidens pilosa L.). Neste caso, tal efeito traduziu-se na redução da 
porcentagem e da velocidade de germinação dessas sementes (Depiné, 2003).

Almeida et al. (2009) constataram que a utilização do extrato de Piper nigrum (L.) empregados em sementes de feijão $V$. unguiculata mantém a germinação e ainda diminui a infestação de insetospragas de sementes armazenadas.

Pode-se concluir, diante do exposto, que o óleo essencial de carqueja não afetou o potencial germinativo das sementes de $V$. unguiculata, independentemente das dosagens e dos métodos utilizados (fumigação ou impregnação).

Neste sentido, o óleo essencial de B. trimera pode ser utilizado em dosagens inferiores a $5 \mu \mathrm{L}$, que não acarretará em comprometimento da qualidade fisiológica das sementes de feijão.

Novas pesquisas devem ser realizadas utilizando-se o óleo essencial de carqueja em dosagens inferiores a $5 \mu \mathrm{L}$, avaliando-se, inclusive, a possível bioatividade sobre insetos-praga de feijão armazenado.

\section{AGRADECIMENTO}

À Unidade Acadêmica de Serra Talhada, da Universidade Federal Rural de Pernambuco, pela disponibilidade da infra-estrutura para realização da pesquisa.

Ao CNPq pelo suporte financeiro ao projeto e pela concessão de bolsas aos autores.

\section{REFERÊNCIA}

ASLAN, I. et al. Toxicity of essential oil vapours to two greenhouse pests, Tetranychus urticae Koch and Bemisia tabaci Genn. Industrial Crops and Products, v.19, n.2, p.167-73, 2004.

ALMEIDA, F.A.C. et al. Viabilidade de sementes de feijão macassar tratadas com extrato vegetal e acondicionadas em dois tipos de embalagens. Acta Scientiarum. Agronomy, v.31, n.2, p.345-51, 2009. BONA, C.M. et al. Carqueja: cultive esta idéia. Curitiba: SEAB-PR, 2002.

CANTERI, M.G. et al. SASM - Agri: sistema para análise e separação de médias em experimentos agrícolas pelos métodos Scoft - Knott, Tukey e Duncan. Revista Brasileira de Agrocomputação, v.1, n.2, p.18-24, 2001.

CARVALHO, N.M.; NAKAGAWA, J. Sementes: Ciência, tecnologia e produção. 4.ed. Jaboticabal: Funep/Unesp, 2000.

CASTRO, H.G.; FERREIRA, F.A. Contribuição ao estudo das plantas medicinais (Baccharis genistelloides). Viçosa: Ed. UFV, 2001.

DEPINÉ, C. Efeito alelopático de carqueja sobre a germinação de plantas daninhas. 2003. 43p. Monografia
(Bacharelado em Ciências Biológicas) - Núcleo de Ciências Biológicas e da Saúde, Centro Universitário Positivo, Curitiba.

EDMOND, J.B.; DRAPALA, W.J. The effects of temperature, sand and soil, and acetone on germination of okra seeds. Proceedings of the American Society for Horticultural Science, v.71, p.428-34, 1958.

FAO. FAOSTAT. Crops. Cow peas, dry. Disponível em: <http://faostat.fao.org/site/567/DesktopDefault. aspx?PagelD=567\#ancor>. Acesso em: 02 set. 2011.

FRITZ, D. et al. Germination and growth inhibitory effects of Hypericum myrianthum and $H$. polyanthemum extracts on Lactuca sativa L. Revista Brasileira de Farmacognosia, v.17, p.44-8, 2007.

FURBECK, S.M. et al. Relationship of seed and germination measurements with resistance to seed weathering cotton. Seed Science and Technology, v.21, n.3, p.505-12, 1993.

LOBATO, A.K.S. et al. Ação do óleo essencial de Piper adunsum $\mathrm{L}$. utilizado como fungicida natural no tratamento de sementes de Vigna unguiculata (L.) Walp. Revista Brasileira de Biociências, v.5, supl.2, p.915-7, 2007.

MACHADO, J.C. Tratamento de sementes no controle de doenças. Lavras: LAPS/ UFLA/ FAEPE. 2000. 138p. MAGUIRE, J.D. Speed of germination-aid in selection and evaluation for seedling emergence and vigor. Crop Science, v.2, n.2, p.176-7, 1962.

MEDEIROS, D.C. et al. Pó de folhas secas e verdes de nim sobre a qualidade das sementes de feijão caupi. Caatinga, v.20, n.2, p.94-9, 2007.

MORS, W.B.; RIZZINI, C.T.; PEREIRA, N.A. Medicinal plants of Brazil. Michigan: Reference Publications Incorporation. 2000. 501p.

PIO-CORREAA, M.P. Dicionário das plantas úteis do Brasil e das exóticas cultivadas. Rio de Janeiro: Imprensa Nacional. 1994. 63p.

SHAAYA, E.M.; KOSTJUKOVSKI, J.; SUKPRAKARN, C. Plant oils as fumigants and contact insecticides for the control as stored-product insect. Journal of Stored Products Research, v.33, p.7-15,1997.

SILVA, K.J.D. Estatística da produção de feijãocaupi, 2009. Disponível em: <http://www.portal doagronegocio.com.br/conteudo. php?id=34241>. Acesso em: 02 set. 2011.

WEAVER, D.K.C.D. et al. nsecticidal actitivy of floral, foliar, and root extracts of Tagetes minuta (Asterales: Asteraceae) against adult Mexican beanweevil (Coleoptera: Bruchidae). Journal of Economic Entomology, v.87, p.1718- 25, 1994a.

WEAVER, D.K.F.V.; DUNKEI, R.C.; POTTER, L. Contact and fumigant efficacy of powdered and intact Ocimum canum Sims (Lamiales:Lamiaceae) against Zabrotes subfasciatus (Bo hemann) adults (Coleoptera : Bruchidae). Journal of Stored Products Research, v.30, p.243-52, 1994b.

WETZEL, M.M.V.S.; RAMOS, S.R.R.; PEREIRA NETO, L.G. Comportamento das sementes de feijâo-caupi no armazenamento a longo prazo. Brasília: Embrapa Recursos Genéticos e Biotecnologia. 2005. 11p. 